\title{
Influencia de la madera juvenil de pino radiata sobre las propiedades físicas y mecánicas de tableros OSB
}

\author{
Influence of radiata pine juvenile wood on the physical and mechanical \\ properties of oriented strand boards (OSB) \\ ROBERT PECHO ${ }^{1}$, RUBEN A. ANANIAS ${ }^{2}$, ALDO BALLERINI ${ }^{2}$, ALAIN CLOUTIER $^{3}$. \\ ${ }^{1}$ Facultad de Recursos Naturales Renovables, Universidad Agraria de la Selva Tingo María, \\ Casilla postal 156, Perú. E-mail: rgpecho2002@hotmail.com \\ 2Universidad del Bío-Bío Chile. E-mail: ananias@ubiobio.cl, aballeri@ubiobio.cl \\ ${ }^{3}$ Université Laval Canadá. E-mail: alain.cloutier@sbf.ulaval.ca
}

\begin{abstract}
SUMMARY
In this work the influence of radiata pine juvenile wood (Pinus radiata D. Don), more specifically the growth ring width and cell wall thickness, on the physical (swelling and linear expansion) and mechanical (internal bond, modulus of rupture, and modulus of elasticity) properties of oriented strand boards (OSB) were analyzed. The wood samples were obtained from 10 trees from a 26-year old, managed plantation in the Eighth Region of Chile. The juvenile wood determination was based on the radial variation of growth ring width measured by image analysis using commercial software. The details of the anatomical structure were similarly determined from microtome samples. The experimental design considered the proportion of juvenile wood $(0 \%, 30 \%, 70 \%$, and $100 \%$ ) and the type of cut of the strands (radial and tangential) to be independent variables. The press cycle involved a maximum temperature of $195{ }^{\circ} \mathrm{C}$, a maximum pressure of 40 bars and a press time of 230 seconds. The results show that the proportion of juvenile wood has an impact on the physical and mechanical properties of OSB. The internal bond (0.46 MPa for juvenile wood and $0.70 \mathrm{MPa}$ for mature wood) and the modulus of elasticity (3271 MPa for juvenile wood and $5159 \mathrm{MPa}$ for mature wood) decreased significantly with an increase in the proportion of juvenile wood. The modulus of rupture varied between $29 \mathrm{MPa}$ for juvenile wood and 36 MPa for mature wood but these values were not significantly different. Thickness swell (32\% for juvenile wood and $24 \%$ for adult wood) was greater than the value specified in the Canadian 0437 series-93 standard. However, the linear expansion $(0.22 \%$ for juvenile wood and $0.17 \%$ for mature wood) met the mechanical standard.
\end{abstract}

Keywords: juvenile wood, mechanical properties, OSB panels, radiata pine.

\section{RESUMEN}

En este trabajo se analizó la influencia de la madera juvenil de pino radiata (Pinus radiata D. Don), en particular el ancho de los anillos de crecimiento y el espesor de la pared celular, sobre las propiedades físicas (hinchamiento y expansión lineal) y mecánicas (adhesión interna, MOR y MOE) de tableros de hojuelas orientadas OSB. La madera para los ensayos fue recogida de 10 árboles en pie de un rodal de 26 años creciendo en plantaciones manejadas de la Octava Región, Chile. La determinación de madera juvenil fue realizada observando la variación radial de los anillos de crecimiento de la madera, sobre la base de análisis de imágenes y el apoyo de un software comercial. Los detalles de la estructura anatómica fueron determinados sobre cortes microtomados, mediante análisis de imágenes usando un software comercial. El diseño experimental consideró como factores variables la proporción de madera juvenil $(0,30,70$ y $100 \%$ de madera juvenil) y el tipo de corte de las hojuelas (radial y tangencial). El ciclo de prensado utilizado consideró una temperatura máxima de $195^{\circ} \mathrm{C}$, una presión máxima de 40 bar y un tiempo de prensado de 230 segundos. Los resultados mostraron que la proporción de madera juvenil condiciona las propiedades físicas y mecánicas de los tableros OSB. El IB (con 0,46 MPa para madera juvenil y 0,70 MPa para madera adulta) y el MOE (madera juvenil de 3.271 y de $5.159 \mathrm{MPa}$ para madera madura) disminuyeron significativamente con el incremento de la proporción de madera juvenil. Los valores del MOR de $29 \mathrm{MPa}$ en madera juvenil y $36 \mathrm{MPa}$ en el caso de madera madura, en cambio, resultaron independientes de la proporción de madera juvenil. El hinchamiento (32\% para 
madera juvenil y $24 \%$ para madera adulta) fue mayor que lo exigido por la norma O437 series 93; en cambio, la expansión lineal (0,22\% para madera juvenil y 0,17\% para madera adulta) cumplió con la norma al igual que todas las propiedades mecánicas estudiadas.

Palabras clave: madera juvenil, propiedades mecánicas, tableros OSB, pino radiata.

\section{INTRODUCCION}

La industria de tableros de hojuelas orientadas (OSB) permite la utilización de diversas especies de madera, no presentando mayores exigencias respecto del diámetro o rectitud de las trozas, pudiendo usarse, además, subproductos del proceso de aserrado tales como cantoneras y tapas. Además, las actuales empresas de OSB en el mundo están incorporando materia prima proveniente de bosques de alta rotación. Esto representa una ventaja ante el contrachapado, su más cercano competidor, que debe esperar un tiempo muy largo de rotación de sus bosques para alcanzar los diámetros que necesita $(1,2)$. La utilización de bosques con alta rotación implica una importante producción en madera juvenil, pero a más bajo costo. La presencia de la madera juvenil adquiere mayor relevancia cuando se trabaja a base de rotaciones cortas, ya que la mayor parte del volumen está constituido por este tipo de madera, o cuando se utiliza en forma integral el árbol $(3,4)$. Entonces, la madera juvenil adquiere una importancia económica en la fabricación de tableros OSB, ya que durante el manejo de un bosque sólo un pequeño porcentaje llega a la edad de la rotación final y la biomasa extraída puede ser destinada a la fabricación de estos tableros OSB, agregando con ello valor a la utilización de la madera juvenil.

Por otra parte, los estudios técnicos han demostrado que las diferencias con respecto a las propiedades de la madera juvenil son a menudo limitadas cuando se utilizan en materiales compuestos, mejorando las variables de fabricación tales como la geometría y la compactación de los constituyentes $(5,6,7,8,10,11,13)$.

En Chile el pino radiata tiene potencialidades para usarse como materia prima en la construcción de tableros OSB, ya que una parte importante de su producción es de madera juvenil. No obstante las investigaciones sobre la influencia de la madera juvenil en las propiedades físicas y mecánicas de los diferentes productos compuestos, es bastante limitada y no describe adecuada- mente su efecto sobre los tableros de hojuelas orientadas.

En estudios sobre madera de Abeto douglas, Wasniewski (14), encontró un $10 \%$ de aumento en el MOE y MOR de tableros de hojuelas al azar distribuidas con el incremento de la edad de 1 a 50 años. Estos mismos parámetros aumentaron de un 30 a $40 \%$ usando subproductos de madera aserrada. Betanzo y Salinas (15) muestran que los tableros OSB confeccionados con madera madura presentaron mejor resistencia al cizalle. Además, Brochman et al. (16) indican que el espesor de las hojuelas mejoró la estabilidad dimensional y las características mecánicas de los tableros OSB. Wasniewski (14) muestra que el efecto de la edad de la madera es notable en la densidad del tablero, en particular en los gradientes de densidad horizontal, que fueron asociados a hojuelas más delgadas y de inferior densidad que el material más joven. Un decrecimiento en la expansión lineal de los tableros de hojuelas al azar, con el incremento de la edad de la madera, fue atribuido al cambio del ángulo fibrilar de pared secundaria. El incremento en el hinchamiento de los tableros con la edad de la madera fue relacionado con diferencias en los parámetros de producción, así como con el espesor de las hojuelas. Igualmente, Betanzo y Salinas (15) indican que los tableros OSB fabricados con madera juvenil fueron más estables dimensionalmente.

En el contexto anterior, los objetivos de este proyecto fueron evaluar el impacto de la madera juvenil (ancho de anillos y espesor de pared celular) en las propiedades físicas (hinchamiento y expansión lineal) y mecánicas (IB, MOR y MOE) de los tableros OSB.

\section{MATERIAL Y METODOS}

Los árboles para el presente estudio proceden de un rodal de Pinus radiata D. Don, de 26 años de edad, de una superficie de 3,8 ha, sometido a un manejo intensivo, ubicado en el fundo 
"El Patagual", comuna de Coronel, perteneciente a una empresa maderera de la VIII Región (Chile). Se recogieron 10 árboles en forma aleatoria bajo criterios de dominancia (dominantes y codominantes), rectitud del fuste (fuste recto) y sanidad (sin presencia de daños). A cada árbol seleccionado se le marcó el lado norte, se midió el diámetro a la altura del pecho (DAP), con una forcípula y se le determinó la dominancia en forma visual. Luego del talado, de cada árbol a la altura del pecho se extrajo una troza de una longitud de $1,30 \mathrm{~m}$ y se midió el diámetro mayor y menor. Enseguida las trozas fueron llevadas al laboratorio Silvotecnológico de la Universidad de Concepción, donde se obtuvieron una pieza central de $50 \mathrm{~mm}$ de espesor y dos semibasas norte y sur. A continuación las dos semibasas fueron llevadas al Pabellón de Tecnología de la Madera (Universidad del Bío-Bío), donde se prepararon las probetas para el estudio de las características anatómicas y las hojuelas necesarias para este trabajo. Adicionalmente una probeta del corte central fue obtenida para la caracterización de los anillos de crecimiento.

La medición de los anillos de crecimiento desde médula a corteza fue realizada mediante análisis de imágenes (scaner) con apoyo de un software comercial (Windendro). Se realizó una medición para cada árbol. La medición del espesor de la pared celular fue realizada en cortes microtomados de la cara transversal, mediante análisis de imágenes con apoyo de un software comercial (Wincell). Por cada árbol se prepararon 20 cortes microtomados, usando técnicas usuales de macerado en agua caliente, tinción con safranina y fijación permanente con bálsamo de Canadá. Se realizaron alrededor de 3.000 mediciones del espesor de la pared celular. Estas mediciones permitieron apoyar la identificación de madera juvenil y adulta.

Una vez caracterizado el material se procede a la preparación de las hojuelas y confección de los tableros OSB, para lo cual cada una de las dos semibasas de cada árbol fueron escuadradas para obtener probetas de madera juvenil (MJ) y de madera adulta (MA), en corte radial (Rd) y tangencial (Tg). La escuadría de las probetas fue determinada de acuerdo a las dimensiones de la hojuelera (55 x $90 \times 150 \mathrm{~mm})$. Enseguida, a partir de estas probetas, fueron obtenidas hojuelas radiales y tangenciales, con las siguientes dimen- siones: espesor de $0,7 \mathrm{~mm}$, ancho 25 a $55 \mathrm{~mm}$ y largo $90 \mathrm{~mm}$. Luego de una clasificación preliminar de las hojuelas, éstas fueron secadas hasta $4 \%$ de contenido de humedad, a una temperatura de $70^{\circ} \mathrm{C}$ por espacio de 24 horas. En este caso el control de la humedad de las hojuelas fue realizado gravimétricamente usando una balanza térmica. A continuación, el encolado fue realizado con la ayuda de una pistola de aire comprimido al interior de la máquina encoladora. Para la formación del colchón se utilizaron cajones formadores debidamente seccionados, la tapa del cajón formador sirvió, además, para aplicar un preprensado a las hojuelas. La primera y tercera capas de las hojuelas (caras) fueron orientadas en forma paralela; en cambio, las hojuelas del centro se ubicaron en forma perpendicular a las caras.

Las especificaciones técnicas consideradas en la fabricación de los tableros OSB fueron las siguientes: razón de encolado: $8 \%$; espesor del tablero: $12 \mathrm{~mm}$ y área del tablero: $0,5 \times 0,5=$ $0,25 \mathrm{~m}^{2}$. Las características técnicas del adhesivo fueron: porcentaje de sólido: $50,5 \%$; viscosidad: $450 \mathrm{cP}$ y pH: 10,96. La conformación del colchón fue de un peso total de $2.000 \mathrm{~g}$ incluido el adhesivo. Las caras se confeccionaron con 35\% del material (700 g cada una) y el centro con un $30 \%$ (600 g). El ciclo de prensado para el presente trabajo fue realizado a base de especificaciones de trabajos anteriores: $195^{\circ} \mathrm{C}$ de temperatura máxima, 40 bar de presión específica máxima y un tiempo de 230 segundos $(17,18)$. Una vez confeccionados los tableros, fueron enfriados a la temperatura ambiente y formateados a la dimensión final de 40 x $40 \mathrm{~cm}$.

El diseño experimental consideró como factores variables la proporción de madera juvenil (4 niveles: $100,70,30$ y $0 \% \mathrm{MJ}$ ) y el tipo de corte (2 niveles: radial y tangencial), resultando una combinación de 32 tableros. Además, se fabricaron 4 tableros testigos (combinación aleatoria de madera juvenil y adulta, y corte radial y tangencial), que se utilizaron como base de referencia para comparar las propiedades de los tableros.

Para estudiar las características anatómicas (ancho de anillos y espesor de pared celular), se consideró un diseño completamente aleatorio. Con respecto al estudio de las propiedades físicas y mecánicas de los tableros OSB, se realizó un análisis de varianza multifactorial de dos factores, o sea, el porcentaje de madera juvenil (\%MJ), 
$100 \% \mathrm{MJ}, 70 \% \mathrm{MJ}, 30 \% \mathrm{MJ}$ y $0 \% \mathrm{MJ}$ y tipo de corte radial (Rd) y tangencial (Tg). Asimismo las diferencias significativas fueron observadas mediante el test de rangos múltiples de Tukey al 95\%.

La evaluación de las propiedades físicas (hinchamiento y expansión lineal) y mecánicas (IB, MOE y MOR) de los tableros OSB se realizaron de acuerdo a la norma canadiense $\mathrm{N}^{\circ} \mathrm{O} 437$ Series-93.

Para el hinchamiento se utilizaron 2 probetas de $150 \times 150 \mathrm{~mm}$, por cada tablero, las que fueron sometidas a inmersión en agua por 2 y 24 horas. El hinchamiento fue calculado a base del cambio en las dimensiones antes y después de la inmersión en agua, usando un micrómetro con una precisión de $0,1 \mathrm{~mm}$ la relación:

$$
H e=\frac{e_{(24)}-e_{(0)}}{e_{(0)}} * 100
$$

Donde:

He = Hinchamiento del espesor después de 24 horas de inmersión en agua fría (\%).

$\mathrm{e}_{(24)}=$ Sumatoria de las 8 medidas del espesor después de 24 horas de inmersión (mm).

$\mathrm{e}_{(0)}=$ Sumatoria de las 8 medidas del espesor antes de la inmersión ( $\mathrm{mm})$.

Para la expansión lineal fue utilizada una probeta de 50x300 mm, por cada tablero, con dimensión longitudinal paralela a las fibras. Las probetas fueron inicialmente secadas en horno hasta $103^{\circ} \mathrm{C}$ por espacio de 24 horas o hasta obtener peso constante. Después del secado, las probetas fueron envueltas en polietileno hasta que se enfriaron a $20^{\circ} \mathrm{C}$. Luego se midió la distancia entre dos puntos distanciados inicialmente a $250 \mathrm{~mm}$. Enseguida las probetas fueran sumergidas en agua a $18^{\circ} \mathrm{C}$ bajo vacío a $92 \pm 6 \mathrm{kPa}(690,1 \mathrm{~mm} \mathrm{Hg})$ por 1 hora, inmediatamente después las probetas fueron sometidas por 2 horas a una presión de $620 \pm 6 \mathrm{kPa}(4.650,4 \mathrm{~mm} \mathrm{Hg})$. Finalizado este periodo se midió nuevamente la distancia entre los dos puntos y la expansión lineal fue calculada a base del cambio en las dimensiones antes y después del ensayo con una precisión de $0,01 \%$, por la relación:

$$
E L=\frac{L_{S}-L_{S h}}{L_{S h}} * 100
$$

Donde:

$E L=$ Expansión lineal de cada panel (\%).

$L_{s} \quad=$ Longitud antes del ensayo vacío-presión $(\mathrm{mm})$.

$L_{s h}=$ Longitud después del ensayo vacío-presión (mm).

Para la adhesión interna IB fueron utilizadas 6 probetas de $50 \times 50 \mathrm{~mm}$, por cada tablero. Las probetas fueron medidas tanto en su espesor, ancho y largo, luego fueron traccionadas desde los extremos de la probeta usando una máquina universal de ensayos mecánicos, capaz de medir con una sensibilidad de $1 \mathrm{~kg}$ y aplicar una carga continua P. El IB se calculó con una aproximación de $0,1 \mathrm{MPa}$, por la relación:

$$
I B=\frac{P_{\operatorname{máx}}}{b^{*} l}
$$

Donde:

$I B$ = Esfuerzo de Tracción Perpendicular (MPa). $P_{\text {máx }}=$ Carga máxima $(\mathrm{kN})$.

$b=$ Ancho de la probeta $(\mathrm{mm})$.

$l=$ Largo de la probeta $(\mathrm{mm})$.

Nota: La velocidad de este ensayo fue de 0,96 $\mathrm{mm} / \mathrm{min}$.

En el caso del MOE y MOR se utilizó una probeta de $50 \times 338 \mathrm{~mm}$, por cada tablero. Las probetas fueron ensayadas, aplicando una carga en el centro del largo usando una máquina de ensayos apropiada capaz de medir y aplicar la carga en forma continua. La velocidad de este ensayo fue de $5,76 \mathrm{~mm} / \mathrm{min}$. El MOR perpendicular fue calculado con una aproximación de 0,1 $\mathrm{MPa}$, por la relación:

$$
M O R=\frac{3 * P_{m a ́ x} * L}{2 * b * e^{2}}
$$

El MOE perpendicular fue calculado con una aproximación de $100 \mathrm{MPa}$, por la relación:

$$
M O E=\frac{L^{3}}{4 * b^{*} e^{3}} * \frac{\Delta \mathrm{P}}{\Delta \mathrm{Y}}
$$

Donde:

$M O R=$ Módulo de Ruptura $(\mathrm{MPa})$.

$M O E$ = Módulo de Elasticidad (MPa).

$\mathrm{P}_{\text {máx }}=$ Carga máxima en la ruptura $(\mathrm{kN})$. 
$b \quad=$ Ancho de la probeta $(\mathrm{mm})$.

$L=\operatorname{Luz} \geq 24 *$ espesor.

$e \quad=$ Espesor de la probeta $(\mathrm{mm})$.

$\Delta P=$ Incremento de la carga (Newton) en la porción lineal de la curva carga v/s deformación.

$\Delta Y=$ Incremento de la deflexión en la distancia media $(\mathrm{mm})$ correspondiente al incremento de la carga $\mathrm{P}$.

\section{RESULTADOS Y DISCUSION}

Caracterización de la madera juvenil (ancho de anillos de crecimiento y espesor de pared celular): El ancho de los anillos de crecimiento y el espesor de la pared celular son presentados en el cuadro 1 . Se observa que la madera juvenil tiene los anillos de crecimiento de madera temprana significativamente más anchos que la madera adulta. En relación con el espesor de la pared celular, las traqueidas de la madera juvenil (MJ) presentan un menor tamaño en el espesor de la pared celular que la madera adulta (MA). Según el análisis estadístico, la madera tardía presenta un significativamente mayor espesor de pared celular que la madera temprana. Este comportamiento es asociado a la diferencias en la actividad cambial en cada periodo vegetativo (10).
El comportamiento anteriormente descrito, o sea, el mayor ancho de anillos y el menor espesor de pared celular de la madera juvenil, permite inferir que durante la fabricación de los tableros la madera juvenil sería mejor compactada, particularmente en las caras cuando sea expuesta a una alta temperatura. Esta hipótesis se fundamenta en la mayor presencia de lignina y menor grado de compactación de la pared celular de la madera temprana $(6,9,10)$.

Los valores del tipo de corte (radial y tangencial) sobre las propiedades físicas de los tableros OSB (hinchamiento y expansión lineal) muestran que el hinchamiento y la expansión lineal en el corte tangencial son mayores respecto al corte radial (cuadro 2).

El tipo de corte presenta diferencias significativas para el hinchamiento en el espesor de los tableros después de 24 horas de inmersión en agua, donde el valor de $\mathrm{p}=0,0011<0,05$. Al realizar el test de rango múltiple de Tukey con $\alpha=0,05$, se encuentra que el hichamiento en los tableros fabricados con hojuelas de corte radial son significativamente mayores que los de corte tangencial. El mayor hinchamiento en los tableros de corte radial es favorecido por la higroexpansión tangencial en el espesor de las hojuelas radiales.

Para los tableros construidos con diferentes porcentajes de madera juvenil, se encuentran di-

\section{CUADRO 1}

Análisis del ancho de los anillos de crecimiento entre madera juvenil y adulta (promedio 10 árboles) y espesor de la pared celular.

Analysis of wide of the ring of growth between juvenile and adult wood (average the 10 trees) and thickness of the cellular wall.

\begin{tabular}{|l|c|c|c|c|c|c|}
\hline \multirow{2}{*}{ Análisis } & \multicolumn{2}{|c|}{ Ancho anillos crecimiento } & \multicolumn{4}{|c|}{ Espesor pared celular (mm) } \\
\cline { 2 - 8 } & M. Juvenil & M. Adulta & \multicolumn{2}{|c|}{ Madera juvenil } & \multicolumn{2}{c|}{ Madera adulta } \\
\cline { 2 - 8 } & & & M. Temprana & M. Tardía & M. Temprana & M. Tardía \\
\hline Media & 9,7 & 4,4 & 0,0041 & 0,0062 & 0,0045 & 0,0084 \\
\hline Tukey & (A) & (B) & (A) & $($ B) & (A) & (B) \\
N & 90 & 110 & 3.000 & 3.000 & 3.000 & 3.000 \\
\hline P valor & \multicolumn{3}{|c|}{0,000} & \multicolumn{2}{|c|}{0,022} & \multicolumn{2}{c|}{0,011} \\
\hline C.V. (\%) & 13,3 & 16,6 & 25,6 & 40,5 & 40,2 & 47,1 \\
Desv. Std. & 1,3 & 0,7 & 0,0010 & 0,0025 & 0,0018 & 0,0039 \\
Mín. & 7,7 & 3,6 & 0,0027 & 0,0043 & 0,0029 & 0,0035 \\
Máx. & 12,2 & 5,5 & 0,0054 & 0,0131 & 0,0073 & 0,0150 \\
\hline
\end{tabular}




\section{CUADRO 2}

Resultados del análisis de varianza de las propiedades físicas de los tableros, según tipo de corte requeridos por norma canadiense O437 Series-93.

Analysis results for variation in the physical properties of boards, according to type of cut required by Canadian norm O437 series- 93 .

\begin{tabular}{|l|c|c|c|c|}
\hline \multirow{2}{*}{ Propiedad } & \multicolumn{2}{|c|}{ Tipo de corte } & \multirow{2}{*}{ Requerido } & \multirow{2}{*}{ Comercial } \\
\cline { 2 - 3 } & Radial & Tangencial & & \\
\hline Hinchamiento (\%) & 25,5 & 29,3 & 15 & 22 \\
Desv. Std. & 5,4 & 2,9 & & \\
\hline Expansión lineal (\%) & 0,18 & 0,22 & 0,35 & 0,25 \\
Desv. Std. & 0,04 & 0,05 & & \\
\hline
\end{tabular}

Fuente: Tablero comercial confeccionado en álamo temblón (19).

ferencias estadísticas significativas para los valores del hinchamiento en el espesor después de 24 horas (cuadro 3), donde el valor de $\mathrm{p}=0,0000$ $<0,05$. Al realizar el test de rango múltiple de Tukey con $\alpha=0,05$, se muestra que los tableros tienen el siguiente comportamiento: el 100\%MJ es diferente a los demás porcentajes de madera juvenil, alcanzando los mayores valores de hinchamiento en el espesor después de 24 horas de inmersión en agua; entre el $70 \% \mathrm{MJ}, 30 \% \mathrm{MJ}$ y $0 \% \mathrm{MJ}$ no presentan diferencias estadísticas significativas exhibiendo un comportamiento más homogéneo, debido probablemente al menor porcentaje de madera juvenil presente en los tableros. La absorción de agua de los tableros resulta mayor a lo especificado por la norma canadiense O437-93. No obstante, se observa que los tableros con pino radiata presentan un menor hinchamiento que los tableros comerciales de álamo (19). El hinchamiento a la escala industrial es, además, reducido usando ceras impermeabilizantes al $1 \%$.

El mayor hinchamiento en los tableros construidos con diferentes porcentajes de madera juvenil es consistente con la presencia de un mayor ancho de anillo de madera temprana y un espesor de pared celular más delgado, los cuales tienen mayores capacidades de adsorber agua, como consecuencia de una menor compactación de la pared celular. Estos resultados coinciden con los reportados en literatura $(8,11,18)$.
El tipo de corte afecta $(\mathrm{p}=0,0189<0,05)$ significativamente la expansión lineal de los tableros, el corte tangencial presenta una expansión lineal significativamente mayor que los tableros con hojuelas radiales. Igualmente, en el cuadro 4 se presenta la expansión lineal de los tableros según la proporción de madera juvenil. De acuerdo al análisis estadístico, el porcentaje de madera juvenil influye significativamente sobre la expansión lineal $(\mathrm{p}=0,0325<0,05)$. De acuerdo al test

\section{CUADRO 3}

Resultados del hinchamiento (\%) en el espesor de los tableros OSB (24 horas).

Results for the swelling thickness of OSB (24 hours).

\begin{tabular}{|l|c|c|c|c|}
\hline Análisis & \multicolumn{4}{|c|}{ Hinchamiento en espesor (\%) } \\
\hline & $100 \%$ MJ & $70 \%$ MJ & $30 \%$ MJ & $0 \%$ MJ \\
\hline Media & 32 & 27 & 26 & 24 \\
Tukey & (A) & (B) & (B) & (b) \\
N & 16 & 16 & 16 & 16 \\
\hline P valor & \multicolumn{5}{|c|}{0,0000} \\
\hline C.V. (\%) & 10 & 15,8 & 14,8 & 20,1 \\
Desv. Std. & 3,3 & 4,3 & 3,9 & 5 \\
Mín. & 28,1 & 20,5 & 20,2 & 18,5 \\
Máx. & 37,4 & 32 & 31,8 & 32,1 \\
\hline
\end{tabular}


de rango múltiple de Tukey con $\alpha=0,05$, se encuentra que los tableros tienen el siguiente comportamiento: entre $100 \% \mathrm{MJ}$ y $70 \% \mathrm{MJ}$, no existen diferencias estadísticas significativas, con valores de expansión lineal más altos; seguido por el $30 \% \mathrm{MJ}$, y finalmente el $0 \% \mathrm{MJ}$ es diferente a todos y es el que alcanza el menor porcentaje de expansión lineal. Al comparar los resultados de la expansión lineal de los tableros construidos con diferentes porcentajes de madera juvenil con el testigo, se observa que no existen diferencias significativas.

Los valores de expansión lineal cumplen lo establecido por la norma, ya que están por debajo del 0,35. Los tableros fabricados con ciento por ciento de madera juvenil (100\% MJ), y sin madera juvenil $(0 \% \mathrm{MJ})$, son los que alcanzan mayor y menor expansión lineal respectivamente. Esto tiene su origen en las características de la estructura anatómica de la madera, particularmente el ancho de los anillos y el espesor de la pared celular. Los resultados de la presente investigación son similares a los reportados en literatura $(8,11,12,14)$.

El efecto del tipo de corte de las hojuelas sobre las propiedades mecánicas de los tableros OSB $(\mathrm{IB} \perp, \mathrm{MOR} \perp \mathrm{y} \mathrm{MOE} \perp)$ construidos con diferentes porcentajes de madera juvenil se encuentran muy por encima de lo requerido por la norma canadiense $\mathrm{N}^{\circ}$ 0437-Series-93 y ligeramente iguales a los valores encontrados a nivel comercial en tableros OSB fabricados con álamo temblón (cuadro 5).

\section{CUADRO 4}

Resultados de la expansión lineal (\%) de los tableros OSB.

Results for the linear expansion of OSB.

\begin{tabular}{|l|c|c|c|c|}
\hline Análisis & \multicolumn{4}{|c|}{ Expansión lineal (\%) } \\
\hline & $100 \% \mathrm{MJ}$ & $70 \% \mathrm{MJ}$ & $30 \% \mathrm{MJ}$ & $0 \% \mathrm{MJ}$ \\
\hline Media & 0,22 & 0,23 & 0,19 & 0,17 \\
Tukey & $(\mathrm{A})$ & $(\mathrm{A})$ & $(\mathrm{AB})$ & $(\mathrm{B})$ \\
N & 8 & 8 & 8 & 8 \\
\hline P valor & \multicolumn{5}{|c|}{0,0189} \\
\hline C.V. (\%) & 23,7 & 24,3 & 12,5 & 21,7 \\
Desv. Std. & 0,05 & 0,06 & 0,02 & 0,04 \\
Mín. & 0,18 & 0,17 & 0,16 & 0,14 \\
Máx. & 0,30 & 0,28 & 0,22 & 0,21 \\
\hline
\end{tabular}

La tracción perpendicular (IB) presenta diferencias estadísticas significativas $(\mathrm{p}=0,000<0,05)$ con el tipo de corte. Según el test de rango múltiple de Tukey al 95\% se observa que los tableros construidos con hojuelas de corte radial presentan los mayores valores de IB respecto al corte tangencial, debido probablemente a un mejor acomodo interno de las hojuelas de corte radial que es favorecido por su estructura anatómica.

La adhesión interna (IB) de los tableros construidos con diferentes porcentajes de madera ju-

\section{CUADRO 5}

Efecto del tipo de corte de las hojuelas en las propiedades mecánicas y los valores requeridos por la norma canadiense CSA O437 Series-93.

Effect of the type of strand cut on the mechanical properties and the values required by Canadian norm O437 series-93.

\begin{tabular}{|l|c|c|c|c|}
\hline \multirow{2}{*}{ Propiedad } & \multicolumn{2}{|c|}{ Tipo de corte } & \multirow{2}{*}{ Requerido } & \multirow{2}{*}{ Comercial } \\
\cline { 2 - 3 } & Radial & Tangencial & & \\
\hline IB (MPa) & 0,666 & 0,532 & 0,345 & 0,48 \\
Desv. Std. & 0,15 & 0,10 & & \\
\hline MOR (MPa) & 38,7 & 30,2 & 12,4 & 16,4 \\
Desv. Std. & 7,6 & 6,1 & & \\
\hline MOE (MPa) & 4.522 & 4.262 & 1.500 & 6.600 \\
Desv. Std. & 821 & 779 & & \\
\hline
\end{tabular}


venil presentan diferencias estadísticas significativas, cuyo valor de $p=0,0000<0,05$. De acuerdo al test de rango múltiple de Tukey con $\alpha=0,05$, se encuentra que la tracción perpendicular de los tableros construidos con el $0 \% \mathrm{MJ}$ es diferente estadísticamente a los demás porcentajes de madera juvenil, su valor de tracción es 0,698 MPa. Entre el $70 \% \mathrm{MJ}$ y $30 \% \mathrm{MJ}$ no existen diferencias estadísticas significativas. Y finalmente el $100 \% \mathrm{MJ}$ es el más bajo, con un valor de 0,464 $\mathrm{MPa}$. En cambio, el tablero testigo alcanza un valor de 0,86 MPa. Cuando la madera juvenil se encuentra en el centro del tablero $(100 \% \mathrm{MJ}$ y $30 \% \mathrm{MJ}$ ) los tableros presentan una mejor cohesión interna (cuadro 6).

Por otra parte, los ensayos de flexión estática son determinados por el módulo de ruptura (MOR) y el módulo de elasticidad (MOE), en la dirección perpendicular de la capa superficial de los tableros. El tipo de corte afecta significativamente al MOR de los $(p=0,0077<0,05)$. Al realizar el test de rango múltiple de Tukey con $\alpha=0,05$, se encuentra que los tableros construidos con hojuelas de corte radial presentan valores más altos que los fabricados con hojuelas de corte tangencial. Probablemente la presencia de los anillos de crecimiento de madera de verano influye de manera más importante en este tipo de corte.

El módulo de ruptura de los tableros construidos con diferentes porcentajes de madera juvenil. De acuerdo al análisis estadístico, éstos no

\section{CUADRO 6}

Resultados de la tracción perpendicular (MPa) de los tableros OSB.

Results for the measurement of the perpendicular traction of OSB.

\begin{tabular}{|l|c|c|c|c|}
\hline Análisis & \multicolumn{4}{|c|}{ Tracción perpendicular (MPa) } \\
\hline & $100 \% \mathrm{MJ}$ & $70 \% \mathrm{MJ}$ & $30 \% \mathrm{MJ}$ & $0 \% \mathrm{MJ}$ \\
\hline Media & 0,46 & 0,65 & 0,58 & 0,70 \\
Tukey & $(\mathrm{A})$ & $(\mathrm{B})$ & $(\mathrm{B})$ & $(\mathrm{B})$ \\
N & 48 & 48 & 48 & 48 \\
\hline P valor & \multicolumn{5}{|c|}{0,0000} \\
\hline C.V. (\%) & 23,1 & 19,4 & 23,2 & 19,1 \\
Desv. Std. & 0,10 & 0,10 & 0,20 & 0,10 \\
Mín. & 0,30 & 0,40 & 0,40 & 0,50 \\
Máx. & 0,70 & 0,90 & 0,90 & 0,90 \\
\hline
\end{tabular}

presentan diferencias estadísticas significativas $(\mathrm{p}=0,0813>0,05)$. Estos valores de ruptura se comportan de manera bien homogénea. Sin embargo, los mayores módulos de ruptura corresponden a los tableros construidos con $70 \% \mathrm{MJ}$, los cuales alcanzan valores de $38,7 \mathrm{MPa}$, y contrariamente los tableros construidos con $100 \% \mathrm{MJ}$ logran sólo 29,3 MPa. El valor del testigo 38,2 MPa es similar a todos los tableros construidos con diferentes porcentajes de madera juvenil. La resistencia máxima de la flexión es recibida en las caras de los tableros, lo que está íntimamente relacionado con la densidad en las caras de los tableros (cuadro 7).

El módulo de elasticidad de los tableros construidos con diferentes porcentajes de madera juvenil demuestra que el tipo de corte no influye significativamente en el MOE ( $p=0,3744>0,05)$. Sin embargo, el tipo de corte radial presenta mayores valores de elasticidad en comparación con los tableros fabricados con hojuelas de corte tangencial. El MOE presenta diferencias estadísticas significativas con la proporción de madera juvenil $(\mathrm{p}=0,0005<0,05)$. Y al realizar el test de rango múltiple de Tukey con $\alpha=0,05$, se encuentra que el módulo de elasticidad de los tableros construidos con el $0 \% \mathrm{MJ}$ y el $70 \% \mathrm{MJ}$ no presenta diferencias estadísticas significativas, y sus valores son los más altos. En cambio, el 30\%MJ es inferior al $0 \% \mathrm{MJ}$ y $70 \% \mathrm{MJ}$, pero es mayor en comparación al $100 \% \mathrm{MJ}$. Y finalmente el $100 \% \mathrm{MJ}$

\section{CUADRO 7}

Resultados del módulo de ruptura (MPa) de los tableros OSB.

Results for the determination of the modulus of rupture of OSB.

\begin{tabular}{|l|c|c|c|c|}
\hline Análisis & \multicolumn{4}{|c|}{ Módulo de ruptura (MPa) } \\
\hline & $100 \%$ MJ & $70 \%$ MJ & $30 \%$ MJ & $0 \%$ MJ \\
\hline Media & 34,4 & 38,7 & 33,4 & 36,2 \\
Tukey & (A) & (A) & (A) & (A) \\
N & 8 & 8 & 8 & 8 \\
\hline P valor & \multicolumn{5}{|c|}{0,0813} \\
\hline C.V. (\%) & 19,9 & 16,4 & 24,1 & 18,9 \\
Desv. Std. & 6,1 & 6,4 & 7,9 & 6,9 \\
Mín. & 23,4 & 33,4 & 25,4 & 28,1 \\
Máx. & 36,9 & 46,3 & 43,9 & 42,9 \\
\hline
\end{tabular}


es el que presenta los menores valores de elasticidad en comparación con los demás tableros construidos con diferentes porcentajes de madera juvenil (cuadro 8).

Comparados los resultados del módulo de elasticidad de los tableros construidos con diferentes porcentajes de madera juvenil con el testigo, se observa que no existen diferencias, siendo estos resultados bien homogéneos. El 0\%MJ y $70 \% \mathrm{MJ}$ presentan mayores valores de elasticidad, probablemente debido a una mejor densificación en la superficie de los tableros. El 70\%MJ presenta madera juvenil en el centro del tablero, mientras que el $0 \% \mathrm{MJ}$ está construido sólo de madera adulta, favoreciendo la flexión de los tableros.

La mejor alternativa del proceso de fabricación de tableros OSB con diferentes porcentajes de madera juvenil es de acuerdo con estos resultados el $70 \% \mathrm{MJ}$, o sea, cuando la madera juvenil es aplicada en las caras y la madera adulta en el centro. A nivel industrial esta combinación sería económicamente ventajosa, porque se estarían empleando árboles de plantaciones de corta rotación, pero habría que realizar modificaciones pertinentes a la formación de dos colchones, o sea, la preparación de dos tipos de hojuelas y la alimentación a través de dos silos diferentes, similar al proceso de fabricación de tableros de partículas, una vía de alimentación de hojuelas de madera juvenil para los colchones de las caras (como las partículas finas) y una vía de alimentación de

\section{CUADRO 8}

Resultados del módulo de elasticidad (MPa) de los tableros OSB.

Results for the determination of the modulus of elasticity of OSB.

\begin{tabular}{|l|r|r|r|r|}
\hline Análisis & \multicolumn{5}{|c|}{ Módulo de elasticidad (MPa) } \\
\hline & $100 \%$ MJ & $70 \%$ MJ & $30 \%$ MJ & $0 \%$ MJ \\
\hline Media & $\begin{array}{r}3.271 \\
\text { (B) }\end{array}$ & $\begin{array}{r}4.907 \\
\text { (A) }\end{array}$ & $\begin{array}{r}4.232 \\
(\mathrm{AB})\end{array}$ & $\begin{array}{r}5.159 \\
(\mathrm{~A})\end{array}$ \\
Tukey & 8 & 8 & 8 & 8 \\
$\mathrm{~N}$ & \multicolumn{5}{|c|}{0,0005} \\
\hline P valor & 20,4 & 19,1 & 19,9 & 14,8 \\
\hline C.V. (\%) & 667,6 & 935,6 & 833,6 & 765,8 \\
Desv. Std. & 2.469 & 3.551 & 3.380 & 4.057 \\
Mín. & 3.955 & 5.583 & 5.300 & 5.760 \\
Máx. & \multicolumn{5}{|c|}{} \\
\hline
\end{tabular}

hojuelas de madera adulta para el colchón central (como las partículas gruesas).

\section{CONCLUSIONES}

Los resultados muestran que el ancho de los anillos y el espesor de la pared celular de la madera condicionan las propiedades físicas y mecánicas de los tableros OSB. En particular el hinchamiento aumenta significativamente con la proporción de madera juvenil, y la tracción perpendicular y flexión estática disminuyen significativamente con la proporción de madera juvenil.

La propiedad física de expansión lineal y las propiedades mecánicas de tracción, $\mathrm{MOR} \perp \mathrm{y}$ MOE $\perp$ de los tableros OSB, construidos con diferentes \%MJ incluido el testigo, superan los valores exigidos por la norma canadiense O437-Series-93. La propiedad física de hinchamiento no cumple con esta norma.

Los tableros que fueron construidos con $70 \%$ de madera juvenil presentan las mejores propiedades físicas y mecánicas; desde el punto de vista de la utilización de madera juvenil, esta combinación sería recomendable de explorar a la escala industrial.

\section{AGRADECIMIENTOS}

Agradecemos al Programa de Magíster en Ciencia y Tecnología de la Madera de la Facultad de Ingeniería de la Universidad del Bío-Bío, por el financiamiento de una parte de este estudio. Al personal del Laboratorio de Materiales Compuestos de la Universidad del Bío-Bío por su apoyo técnico, y al Laboratorio Silvotecnológico de la Universidad de Concepción por la donación de la madera utilizada en este estudio.

\section{BIBLIOGRAFIA}

(1) FAO. Tableros contrachapados y otros paneles a base de madera. Organización de las Naciones Unidas para la Agricultura y la Alimentación, Roma. 1968, 250 p.

(2) ROFFAEL, E., T. SCHNEIDER. Investigation on partial subtitution of strands in oriented strand boards (OSB) by different lignocellulosic raw materials. Institute for Wood Biology and Wood Technology. Georg August University of Göttingen, Busgenweg. 2003,16 p.

(3) DELMASTRO, R., J. DIAZ-VAZ, J. SCHLATTER. Variabilidad de las características técnicas hereditarias 
del Pinus radiata D. Don, revisión bibliográfica, Investigación y Desarrollo Forestal, 1980, Documento de Trabajo (34), $42 \mathrm{p}$.

(4) ZOBEL, B., J. VAN BIUJTENEN. Wood variation its causes and control, Springer Series in Wood Science, New York. Cap. Número 3 y 5.1989, p.18-40.

(5) BAILLERES, H., B. CHANSON, B., M. FOURNIER-DJIMBI. Plantaciones de árboles de calidad y de rápido crecimiento de productos forestales en los trópicos. Vol. 3, TEMA 12. August 26-31, 1996, IUFRO WP S5: 01-04.

(6) DIAZ-VAZ, J. Anatomía de maderas. Facultad de Ciencias Forestales. Universidad Austral de Chile. Valdivia, Chile. $2003.151 \mathrm{p}$.

(7) DIMITRI, L., C. BISMARCK, P. BOTTCHER. J. SCHULZE. Production and use of poplar small-wood particleboard manufacture. Holzzucht, 1981, vol. 35, $\mathrm{N}^{\circ}$ (1/2):, p. 1-7.

(8) GEIMER, R., J. CRIST. Structural flakeboard from shortrotation intensively cultured hybrid Populus clones. Forest Products Journal, 1980, vol. 36, N ${ }^{\circ}$ 6, p. 42-48.

(9) LARSON, P., D. KRETCHMANN, A. CLARK, J. ISEBRANDS. Formation and properties of juvenile wood in southern pines: A Sinopsis. Forest Products Laboratory. 2001, $42 \mathrm{p}$.

(10) PANSHIN, A., C. DE ZEEUW. Textbook of wood technology. Fourth ed. Editorial McGraw-Hill Book Company. 1980, 722 p.

(11) PUGEL, A., E. PRICE, C. HSE. Composites from southern pine juvenile wood. Part I, Panel fabrication and initial properties. Forest Products Journal, 1990, vol. 4, $\mathrm{N}^{\mathrm{o}}$ 1, p. 29-33.

(12) PUGEL, A., E. PRICE, C. HSE, T. SHUPE. Composites from southern pine juvenile wood. Part 3, Juvenile and mature wood furnish mixtures. Forest Products Journal, 2004 , vol. $54, \mathrm{~N}^{\circ} 1$, p. 47-52.

(13) STEFANIAK, J. Use of juvenile wood in production of particleboard: Properties of particleboard produced from pine branch wood. Prace Komiisji Technol. Drewna, 1981, vol. 10, p. 95-116.

(14) WASNIEWSKI, J. Evaluation of juvenile wood and its effect on douglas fir structural composite panel. In: Proc. $23^{\text {rd }}$ Particleboard and Composite Materials Symposium, Washington State University. 1989, 16 p.

(15) BETANZO, K., F. SALINAS. Estudio del comportamiento de elementos combinados de madera juvenil y madura de Pino insigne (Pinus radiata D. Don). Seminario de Titulación Ingeniería de Ejecución en Maderas UBB. Concepción, Chile, 1998, 96 p.

(16) BROCHMAN, J., C. EDWARDSON, R. SHMULSKY. Influence of resin type and flake thickness on properties of OSB. Forest Products Journal, 2004, vol. 54, № 3, p. 51-55.

(17) FREIRE, L. Evaluación de ciclos de prensados en la fabricación de tableros OSB-02 de álamo y pino. Seminario de Titulación Ingeniería de Ejecución en Maderas. UBB. Concepción, Chile, 2002, 104 p.

(18) PINO, L. Evaluación de tres especies: pino insigne ( $P i$ nus radiata D. Don), coigüe (Nothofagus dombeyi), roble (Nothofagus obliqua) en la fabricación de tableros Oriented Strand Board tipo 02. Seminario de Titulación Ingeniería de Ejecución en Maderas. UBB. Concepción, Chile, 2002, $130 \mathrm{p}$.

(19) CODINA, M. Determinación de las propiedades mecánicas, físicas y de unión del tablero estructural Oriented Strand Board (OSB), Tesis de licenciatura en Cs. Forestales, Talca, Chile, 1998, 77 p. 